\title{
Sexual Health Frameworks
}

Stephanie Baird, LMHC

\section{LEARNING OBJECTIVES}

- EMDR clinicians gain an introductory understanding of modern sexual health.

- EMDR clinicians learn about factors interfering with discussing sexuality and increase their own comfort with addressing sexual health.

- EMDR clinicians explore their own Sexological Worldview.

- EMDR clinicians fully define consent and the PLISSIT (Permission, Limited Information, Specific Suggestions, Intensive Therapy) model of sexual health communication.

- EMDR clinicians learn about several positive sexual health models (OWL, Planned Parenthood, 4-D Wheel, Global Declaration of Sexual Rights and pleasure).

- EMDR clinicians will begin integrating sexual heath information into the EMDR Adaptive Information Processing (AIP) model. 


\section{INTRODUCTION TO SEXUAL HEALTH}

Congratulations on taking this next step to empower yourself and your clients toward full healthy sexual functioning. If you are like me, and most Western psychotherapists, your education and background included very little information about sexual health. Perhaps you were able to take one class on human sexuality in graduate school; maybe you were lucky enough to enjoy two classes. Most of us received zero to just 6 hours of basic sex education in our grade school, and depending on the decade and geographic region of our middle school, our education likely only mentioned menstruation and abstinence.

Even pursuing advanced specialization and/or certification in eye movement desensitization and reprocessing (EMDR) therapy, a therapy that every day helps thousands of folks overcome sexual (and other) trauma, does not bring automatic access to sex-positive information. Additionally, the statistics regarding sexual assault remain sobering. One in three ciswomen, one in four cismen, and two out of three transgender individuals will experience sexual assault in their lifetimes, much of it by the age of 18 (National Center for Injury Prevention and Control, Division of Violence Prevention, 2021).

Over the years I have learned that the majority of clients seeking psychotherapy have a history of sexual trauma and find this to be true in my own private practice. These high statistics informed my drive to become a trauma-focused EMDR therapist and now inform my drive to integrate and share positive sexual health information, resources, and healing in our work with survivors. This chapter provides a foundation of sexual health, introducing you to the several uplifting paradigms.

\section{What Is "Sexual Health"?}

The World Health Organization (WHO, n.d.) defines sexual health as "a state of physical, emotional, mental, and social well-being related to sexuality: it is not merely the absence of disease, dysfunction, or infirmity. Sexual health requires a positive and respectful approach to sexuality and sexual relationships, as well as the possibility of heaving pleasurable and safe sexual experiences, free of coercion, discrimination, and violence. For sexual health to be attained, the sexual rights of all persons must be respected, protected, and fulfilled."

The Office of the Surgeon General and Office of Population Affairs (2001) defines sexual health as "inextricably bound to both physical and mental health. Just as physical and mental health problems can contribute to sexual dysfunction and disease, those dysfunctions and diseases can contribute to physical and mental health problems. Sexual health is not limited to the absence of disease or dysfunction, nor is its importance (sic) confined to just the reproductive years ... It includes freedom from sexual abuse and discrimination and the ability to integrate their sexuality into their lives, derive pleasure from it, and to reproduce (if one wishes)." 


\section{Working Definition of Sex and Sexual Activity}

For the purposes of this book, sex and sexual activity may refer to any behavior that involves any amount of sexual interest/desire, and/or arousal among any configuration of consenting individuals and genders. This non-exhaustive list of behaviors includes kissing, deep kissing, making out, outercourse (any nonpenetrative activity), touching sensitive and/or erogenous areas like breasts and genitals, erotic/sensual massage, masturbation, solo or mutual masturbation, giving and/or receiving oral sex, penis-vagina intercourse, anal sex, manual stimulation, consenting adult kink/bondage and discipline, dominance and submission, sadism, masochism (BDSM) activities, and/or use of sex toys with any consenting gender and/or genitalia configuration. And while individuals and organizations have been studying sex, sexuality, and sexual health for decades, in my work with clients I often come back to Peggy Kleinplatz's direct and arresting research question "What kind of sex is worth wanting?" (2009).

\section{DECLARATION OF SEXUAL RIGHTS AND PLEASURE}

Since 1978 the World Association for Sexual Health (WAS) has worked towards sexual health and rights. Thousands of members attend global biennial congresses and in recent years have made declarations for sexual rights.

\section{Declaration of Sexual Rights}

This is adapted from the the World Association for Sexual Health (WAS). (2014, March). Declaration of Sexual Rights. Retrieved from https://worldsexualhealth .net/wp-content/uploads/2013/08/declaration_of_sexual_rights_sep03_2014. pdf.

1. The right to equality and freedom of sexual expression.

2. The right to make decisions about one's body in regard to sexuality.

3. The right to be safe from all forms of sexual violence and coercion, and be free from inhumane treatment or punishment because of one's sexual or gender expression.

4. The right to accurate scientific information and education about sexuality and sexual health.

5. The right to freely create and dissolve marital and other similar relationships.

6. The right to family planning resources.

7. The right to organize freely to promote sexual rights and health.

8. The right to privacy regarding sexual matters.

9. The right to judicious laws and remedies regarding sexual rights.

10. The right to the highest attainable standard of sexual health, including sexual safe and pleasurable sexual experiences. 


\section{Declaration on Sexual Pleasure}

At WAS's congress in Mexico City (October 2019) they improved their previous declaration by adding a separate declaration on sexual pleasure adapted from a definition from the Global Advisory Board for Sexual Health and Wellbeing (GAB, 2016)

Sexual pleasure is the physical and/or psychological satisfaction and enjoyment derived from shared or solitary erotic experiences, including thoughts...dreams, [and autoeroticism]. Self-determination, consent, safety, privacy, confidence and the ability to communicate and negotiate sexual relations are key... factors for pleasure to contribute to sexual health and well-being. Sexual pleasure should be exercised within the context of sexual rights...[including] equality and non-discrimination, autonomy and bodily integrity, and the right to the highest...standard of health and freedom of expression. The experiences of human sexual pleasure are diverse and sexual rights ensure that pleasure is a positive experience for all concerned and not obtained by violating... human rights and well-being. $(G A B, 2016)$

Some of WAS's declaration includes:

- Sexual pleasure is a fundamental part of sexual and human rights and includes the possibility of diverse sexual experiences.

- Access to appropriate sources of sexual pleasure is part of human well-being.

- Sexual health and well-being include pleasurable and safe sexual experiences free of discrimination, coercion, and violence.

- Sexual pleasure shall be globally integrated into education, health services, research, and advocacy and contributes to global health.

I list these various sexual guidelines and declarations to emphasize the importance of including sexual health as a vital and essential piece of our clients' healing and lives. Van der Hart and others (2006) often describe healing from trauma as a three-phase comprehensive approach. Phase One involves stabilization (resourcing, grounding, etc.). Phase Two includes the nitty-gritty of treatment (including EMDR therapy Phases 3-8). Phase Three of overall trauma treatment includes improving intimacy, sexual health, and self-actualization.

If we all had been lucky enough to receive sex education reflecting WAS values (as well as the OWL and Planned Parenthood values mentioned later in this chapter), from early childhood, I believe the sexual trauma statistics would be greatly reduced and all of us would happily exist in "Phase Three" of overall treatment: empowered and fulfilling sexual health and intimacy. 


\section{CULTURAL AND INDIVIDUAL FACTORS INTERFERING WITH BRINGING SEXUAL HEALTH INTO THE EMDR THERAPY OFFICE}

Despite most of us walking by magazine covers at the grocery store touting advice like "fifty ways to spice up your sex life" or "achieve your most powerful orgasm yet," our culture as a whole remains erotophobic, shaming, and "puritanical" when it comes to embracing sexual pleasure and health. "Erotophobia" is a term used by sex educators Rosalyn Dischavio (2016) and Marty Klein (2010), among others, to refer to our society's fear of anything sexual.

We have now had several decades of sex-positive activism, ramping up in the 1960s with the sexual revolution and burgeoning forms of birth control, continuing through the second wave liberation-oriented feminism of the seventies, surfacing again in the nineties with third wave feminism (partially in response to right-wing Christian/Reaganomic backlash in the eighties and the rise and stigmatization of HIV/AIDS). The nineties' third wave feminism increased public awareness of sexual assault and emphasized intersectionality of race, culture, class, gender, sexual orientation, and so forth. The nineties also brought an army of sex-positive feminists activists like Susie Bright (sex-positive essayist and author of Sexual State of the Union [1997]), Carol Queen (popular sex educator, adult toy store co-owner, and author of Real Live Nude Girl [2002]), and Tristan Taormino (writer, activist, filmmaker), along with the mainstreaming of feminist sex toy stores like Good Vibrations in California. Safer sex discussions also increased in response to the HIV/AIDS crisis. Much of the mainstream can thank Dr. Ruth Westheimer (2012) for all of her trailblazing work candidly and openly tackling any sexual matter thrown her way, from the 1980s to the present.

Increasingly available internet pornography burst onto the scene in the late 1990s and early 2000s, single-handedly misinforming millions of individuals about average sizes of body parts and once again locking folks into stereotypes of masculine individuals actively seeking and receiving sex, and feminine individuals "happy to oblige" others' desires, passively neglecting their own. The widespread availability of e-readers plus steamy erotic fanfiction (e.g., the problematic Fifty Shades of Grey published in 2011) allowed feminine individuals to catch up with masculine individuals in viewing and engaging with erotic material.

The \#metoo movement is one of our most recent cultural sexual history developments. Initially beginning in 2006 when Black, Indigenous, and People of Color (BIPOC) sexual assault survivor and activist Tarana Burke developed the phrase, it erupted in 2017 when actress Ashley Judd accused Harvey Weinstein of sexual assaulting her. This accusation soon unleashed a torrent of disclosures from other famous and non-famous ciswomen (and some cismen) against (mostly) cismen in power. The \#metoo movement has also brought with it much needed discussion of consent, which will be discussed in more detail shortly. 
However, despite this ever-widening spiral of positive sexual health awareness, our puritanical and shaming culture is not going out without a fight. This means that despite the availability of more sex-positive television shows such as Mrs. Fletcher (HBO), Sex Education, and Bridgerton (Netflix), several factors continue to interfere with psychotherapists (even EMDR therapists who regularly discuss sexual trauma) addressing positive sexual health with clients. Some of these factors include therapists' own discomfort in discussing sexuality, therapists' own unexplored/unresolved issues regarding sexuality, and/or therapists' own traumatic histories or propensity for vicarious traumatization (Saakvitne \& Pearlman, 1996).

Even if we feel comfortable and confident discussing sexual health, we may harbor uncertainty about our clients' own comfort levels and interest, worrying that introducing sexual health material might make our clients uncomfortable. Other concerns for us may include perceived boundary issues, potential lawsuits, potential attraction/arousal, our own appropriate versus inappropriate curiosity, misspeaking a term, or introducing a topic on which we have less expertise. However, according to Kevin Gallagher (2014), overall research shows that many clients want to bring up and discuss sexual concerns, yet most prefer their providers inquire first. Gallagher noted that, in fact, most clients rarely ever bring up their concern at all, likely due to concerns about whether they are "normal." In 2020 Klein noted that a typical view of "normal" sexuality includes: "sex when tired, too high or too low expectations, and awkwardness and self-consciousness," versus a joyful, fulfilling, and positive part of life.

Bringing sexual health into our intakes and conversations with our EMDR clients not only gives them likely much-needed permission to mention sexual concerns, it also combats erotophobia in our society at large. In turn our clients will be able to embrace a personal vision of their sexuality that goes beyond "normal," "typical," or "average" into the joyful and pleasurable extraordinary.

Finally, while perusing the Psychology Today therapist directory listings in 2020 , sex therapy was not available as a psychotherapy focus/topic (thankfully EMDR is an option). As of May 2021, I am happy to report sex therapy is now an option.

\section{CONSENT}

The concept of consent is relevant in all ways as we continue on our journey toward integrating sexual health. Hopefully you are enthusiastically consenting to read and integrate the information in this book. As we work with clients, particularly utilizing EMDR therapy, we ideally constantly ask for and obtain consent as we move through the EMDR eight stages. The \#metoo movement has helped generate increased and essential discussion about sexual consent. 
As someone who came of age in the eighties and nineties, not much was discussed or even known about active negotiation of sex-related activities. During my work in the late nineties, primarily working with sexual assault survivors, consent concepts emerged in clinical work as well in rape crisis educational campaigns. However, most of these campaigns centered around the "no means no" concept. This, of course, is paramount. Humans being able to say no to unwanted activities is the foundation from which we can later say yes to wanted activities.

Much of the twentieth century stereotypical cismale and cisfemale gender expression involved the idea that a cisgender man should always want and try for sex, and that a "good" cisgender woman should be a "gatekeeper", saying no often, but eventually "giving in" (like Scarlet O'Hara and Rhett Butler in "Gone With the Wind"- he forcibly dragged her, kicking and fighting, upstairs for sex, and then the next scene shows her post-sex, looking content). It wasn't okay for a woman to outright want and ask for sexual activity - that desire somehow put the woman in a "slut" category. Luckily our wonderful twenty-first century has brought increased activism with folks naming "slut-shaming" as a bad thing, along with the creation of the problematic term "man-slut" (which I dislike), in an attempt to equalize notions of sexual desire.

Now that we are one-fifth into the twenty-first century, with \#metoo solidly in place, humans thankfully continue to expand the conversation around consent. At a minimum, for a person to legally consent to any sexual activity (physical, verbal, or otherwise), the person needs to be of age (16 is the youngest age for any type of intercourse in Massachusetts; this varies state by state).

Consent has further evolved into the acronym FRIES. According to the Planned Parenthood website (https://www.plannedparenthood.org/learn/relationships/sexual-consent), modern full consent is:

1. Freely given. "Consenting is a choice you make without pressure, manipulation, or under influence of drugs or alcohol."

2. Reversible. "Anyone can change their mind about what they feel like doing, anytime. Even if you've done it before" and you are preparing to do it again.

3. Informed. "You can only consent if you have the full story. For example, if someone says they'll use a condom, but don't, then there isn't full consent."

4. Enthusiastic. "When it comes to sex, you should only do things you WANT to do, not things you are expected to do."

5. Specific. "Saying yes to one thing (like going to the bedroom to make out) doesn't mean you've said yes to other" things (like intercourse).

I personally love the addition of enthusiasm, as this concept finally equalizes desire within all the genders. No longer does sex have to reflect the Western stereotype of a cisman being socialized all his life to seize any opportunity of sex (a concept resented by many cismen and others). Now women and other genders can seize the permission, and the imperative, to enthusiastically ask for what they want. 


\section{INCREASING EMDR THERAPIST COMFORT WITH ADDRESSING SEXUAL HEALTH}

Two of the best ways to increase one's comfort level with addressing sexuality involve self-reflection (examining one's own biases and stereotypes) and continuously seeking information and education. Reading this book, if this is your first foray into accurate and empowering sexual health, is a great step toward education. This book mentions many more resources that will hopefully find its way onto your office bookshelf or bookmarked on your web browser. Stephanie Buehler's indispenable primer What Every Mental Health Professional Needs to Know About Sex, Third Edition (2021) recommends topics for the professional to journal at the end of each chapter, another wonderful step toward self-reflection and sexual health acclimation.

There are many organizations and individuals that offer specific training and workshops on sexual health. The national organization that addresses sex education and counseling is the American Association of Sexuality Educators, Counselors and Therapists (AASECT). For anyone wishing to pursue further sexuality certification, taking a three-day SAR (sexual attitude reassessment) class is required. SARs are designed to "inundate participants with sexual material in order to bring awareness to areas of discomfort or bias, and through exploration and discussion, ease participants into greater comfort and confidence in approaching this work" (South Shore Sexual Health Center, n.d.). I highly recommend signing up for a SAR class if you wish to expand your comfort with sexuality-related concerns. Not only will you learn about every imaginable sexual topic, often through videos, guest speakers/panels, interactive learning, and relevant readings, you will meet a group of open-minded folks also willing to explore the outer limits of current societal norms. Completing a SAR will tackle both the self-reflection and educational piece, as well.

Another very effective strategy, one that I greatly benefited from in the years leading up to writing this book, includes receiving regular consultation or supervision regarding your sexuality-related cases. I was fortunate to gather monthly with a small group of clinicians meeting at a sex therapy practice. These meetings provided education, validation, and space for reflection on bias and discomfort, as well as internalizing and integrating many of the concepts you will be reading further about in this book.

\section{THERAPIST'S SEXOLOGICAL WORLD VIEW}

A Sexological Worldview (Sitron \& Dyson, 2012) is the "result of the socialization process that is comprised of values, beliefs, opinions, attitudes, and concepts specific to sexuality, including any and all sexual behavior and identities." This socialization comes from television, films, books, schools, the news, media, 
social media, pornography, religion, peers, and family, and so on. As therapists it is crucial that we continuously examine our own worldviews, remain aware that clients may not inhabit our worldview, and set aside our own biases in order to best help our clients. Our goals for ourselves, and ultimately our clients, include moving away from a rigid right or wrong dualistic way of thinking, toward a more relativistic evolution of a changing, progressing world, with room for greater sexual diversity and acceptance of others' realties. This larger worldview helps provide the foundation for a culturally competent sexual health practice. Additionally, embracing the concept of polysemicity can help us attain this greater worldview. Poylsemicity essentially means that someone else's experiences may not be what ours would be, even if we do the exact same thing (Klein, 2010). For example, two cismen can report very different experiences regarding the exact same sexual activity.

\section{PLISSIT MODEL OF ADDRESSING SEXUAL HEALTH}

One of the first concepts I learned in my foray into sexual health involves the PLISSIT model developed by Annon in 1976. The PLISSIT initials stand for Permission, Limited Information, Specific Suggestions, and Intensive Therapy.

Permission is considered a first level intervention in which the therapist provides permission for the client to discuss/talk/ask questions and/or disclose client feelings and concerns related to specific sexual health issues. "By encouraging clients to share their thoughts, questions, etc., you validate the issue as a legitimate health matter" (Annon, 1976). This may be the most important step and help we can provide certain clients that have been searching for answers or validation for years. You can explicitly provide permission by asking clients verbally or in your intake paperwork if they have any sexuality-related concerns. You can also make mention of your experience and comfort with sexual health on your website or listings. By having sex-positive posters, books, or pamphlets displayed in your waiting room and office, you can provide an environment that says "we can talk about sex here."

Limited Information is a second level intervention, goes beyond the permission obtained in first step, and provides basic information about the topic. During this step you might provide handouts, dispel myths and preconceptions, and refer clients to books and websites. This book references many educational tools and resources you can add to your library of knowledge.

Specific Suggestions comprises the third level and involves advanced level of knowledge or specific expertise. If you are not comfortable or able to provide these, and the client continues to report sexual difficulty, even after accomplishing all the relevant EMDR therapy targets (sexuality-related included), you may need to refer your client to a certified sex therapist, pelvic floor physical therapist, midwife or sex-positive ob/gyn, or other sexual health specialist. 
The fourth level intervention of Intensive Therapy (if needed) is typically provided by an outside specialist (e.g., sex therapist or pelvic floor physical therapist) for a particular issue, such as continued pelvic pain or erectile dysfunction.

\section{POSITIVE MODELS OF SEXUAL HEALTH}

Since being on this journey of integrating sexual health into my own EMDR practice, I have discovered many wonderfully positive models of sexual health. My favorites are mentioned in this section.

\section{Our Whole Lives Sexuality Education Curriculum}

I fell in love with Our Whole Lives (OWL) after my local Unitarian Universalist (UU) congregation asked me if I wanted to attend a weekend training for seventh through twelfth grade curriculum in 2018 , with the intent of volunteering to teach the 9-month long curriculum to our congregational youth. I jumped at this lifelong chance to proactively prevent sexual trauma by inoculating youth (and later, adults) with as much factual, educational, and sex-positive information as the curriculum could provide.

The concepts and guidelines that inform this curriculum grew from the research of the Sexuality Education Task Force in the early nineties and from the work of many educators and UU and UCC (United Church of Christ) congregations who saw a lack of evidence and factual-based sexuality education for youth, developing curriculum for the life-span. You don't have to be a UU or UCC member to receive training to teach the various OWL curricula (broken into these age ranges: Kindergarten through First Grade, Fourth through Sixth Grade, Seventh through Ninth Grade, Tenth through Twelfth Grade, Young Adult, Adult, and now Older Adult). Like taking a SAR, becoming OWL trained is a good way to open up one's mind and sexological worldview.

Keeping in mind these OWL program values, assumptions, and circles of sexuality makes excellent guidelines for a sex-positive therapy practice.

\section{OWL Program Values}

Self-Worth: "Every person is entitled to dignity and self-worth, and to their own attitudes and beliefs about sexuality" (Wilson, 2014).

Sexual Health: "Knowledge about human sexuality is helpful, not harmful. Every person has the right to accurate information" (Wilson, 2014). Healthy sexual relationships are consensual, non-exploitative, mutually pleasurable, safe (low or no risk of sexually transmitted infections (STIs), emotional pain, unintended pregnancy), developmentally appropriate, based on mutual expectations and caring, respectful. Any type of sexual intercourse is only one of the many valid ways of expressing sexual feelings with a partner. 
Responsibility: We enrich our lives by expressing sexuality in ways that enhance human wholeness and fulfillment and can express love and pleasure. "All persons have the right and obligation to make responsible sexual choices" (Wilson, 2014).

Justice and Inclusivity: "People of all ages, sexual identities, races, ethnicities, genders, income levels, abilities, and sexual orientations must have equal value and rights. All of the following are natural in the range of human sexual experience: being romantically and sexually attracted to more than one gender (bisexual), the same gender (homosexual), another gender (heterosexual), and/or to those with a more fluid understanding of their own and others' gender (pansexual), and experiencing no sexual attraction (asexual)" (Wilson, 2014). There should be no coercion or exploitation in sexual relationships, nor should humans be subjected to double standards or stereotypes.

\section{OWL Assumptions}

It can be helpful to hold these OWL assumptions in mind:

"All humans are potentially sexual beings from before birth until death. It is natural to express sexual feelings in a variety of ways. Sexuality is a good part of the human experience and includes much more than sexual behavior. People engage in healthy sexual behavior for a variety of reasons including to express caring and love, experience intimacy and connection with another, share pleasure, bring new life into the world, and have fun and relax. Sexuality in our society is damaged by violence, exploitation, alienation, dishonesty, abuse of power, and the treatment of persons as objects. It is healthier for young teens to postpone forms of intercourse" (Wilson, 2014).

\section{OWL Circles of Sexuality}

This diagram adapted by OWL depicts the overlapping and intersecting circles of sexuality, which permeate human experience, according to OWL (see Figure 1.1). Dennis Dailey originally developed this for a youth education curriculum in 1995. It remains a solid framework from which to explore sexuality.

\section{Planned Parenthood Model of Sexual Health}

Planned Parenthood sex educators base their curriculum on material developed by ETR (Education, Training and Research) whose goal is to advance to health equity. ETR's textbook Sexuality Education: Theory and Practice (Bruess \& Schroeder, 2018) provides a wealth of history and information regarding sexuality education and lists several major guidelines for comprehensive sexuality education such as the United Nations Educational, Scientific and Cultural Organizations' (UNESCO) and the Sexuality Information and Education Council of the United 
Sexuality encompasses nearly every aspect of our being, from attitudes and values to feelings and experiences. It is influenced by the individual, family, culture, religion/spirituality, laws, professions, institutions, science and politics.



FIGURE 1.1 Circles of Sexuality.

Source: Adapted from Life Planning Education, 1995, Advocates for Youth, Washington, DC, Advocatesforyouth.org, based on the original work of Dennis Dailey, Professor Emeritus, University of Kansas.

States' (SIECUS) guidelines as helpful psychoeducation. We can share these with our clients, pointing out that most of us never received any sex education at all, let alone comprehensive sex education adhering to these fairly extensive guidelines. Acknowledging the global and pervasive lack of helpful sex education can reduce our clients' sense of shame or inadequacy around any sexual health gaps they may posses.

UNESCO's (2018) sexuality education goals are "to equip children and young people with knowledge, skills, attitudes and values that will empower them to realize their health, well-being, and dignity; develop respectful social and sexual relationships; consider how their choices affect their own sexual well-being and that of others; and understand and ensure the protection of their rights throughout their lives." UNESCO advocates for both formal and non-formal settings, both in-school and out-of-school settings and includes scientifically accurate information, incremental (building through development), age and developmentally appropriate, based on a curriculum, inclusive of human rights, and culturally appropriate and relevant. 
SIECUS (2004) lists four main goals of sexuality education: (a) provide accurate information about human sexuality, (b) provide an opportunity for people to develop and understand their values, attitudes, and insights about sexuality, (c) help people develop relationships and interpersonal skills, and (d) help people exercise responsibility with regard to sexual relationships (including abstinence, contraception use, and coping with sexual pressure).

SIECUS (2004) also includes thirty characteristics of sexually healthy adults. Here are just a few: appreciate one's own body, interact with all genders in respectful ways, express love and intimacy in appropriate ways, avoid exploitative or manipulative relationships, take responsibility for one's own behavior, enjoy and express one's sexuality throughout one's life, reject sexual stereotypes and promote acceptance of diverse sexualities.

\section{GINA OGDEN'S 4-DIMENSIONAL WHEEL OF SEXUAL HEALTH}

Gina Ogden has developed a wonderfully comprehensive and holistic approach to sexual health, worth learning about briefly here and more on your own as this approach links well with EMDR therapy concepts. Ogden's 4-Dimensional Wheel of sexual health consists of four quadrants: Physical, Mental, Emotional, and Spiritual, similar to EMDR's three-prong approach of body, mind, and emotions. See Figure 1.2.

The Physical quadrant involves the person's range of sensory experience (smell, touch, taste, sight, hearing), movement and stillness, comfort and safety, arousal, orgasm, and other physical pleasures/sensations. This quadrant can be characterized by heightened senses. The "shadow side" can contain pain, disgust, numbness, and dysfunction (Ogden, 2016).

The Emotional quadrant contains the person's range of feelings (love, passion, yearning, anger, hatred, and fear), empathy, self-compassion (described by Dalai Lama as the ability to "love yourself and others no matter how conflicted your feelings may be"), trust (the ability to let go of control), open-heartedness, and heightened feelings. The shadow side can include disappointment, anxiety, depression, restriction, anger, boredom, shame, and guardedness (Ogden, 2016).

The Mental quadrant includes beliefs and messages about sexuality and spirituality, including religious messages. Ogden also includes imagination, intuition, memory, dreams, waking dreams and fantasies, along with wishes, intentions, anticipations, and expectations. This quadrant can involve a curious open-mind, problem-solving, increased understanding, and expanded beliefs. The shadow side may contain negative messages and rigid attitudes about what sex should be like (Ogden, 2016)

The Spiritual quadrant involves a deep sense of connection with one's self, partner(s), and/or "higher power." It can include inner visions, communication 


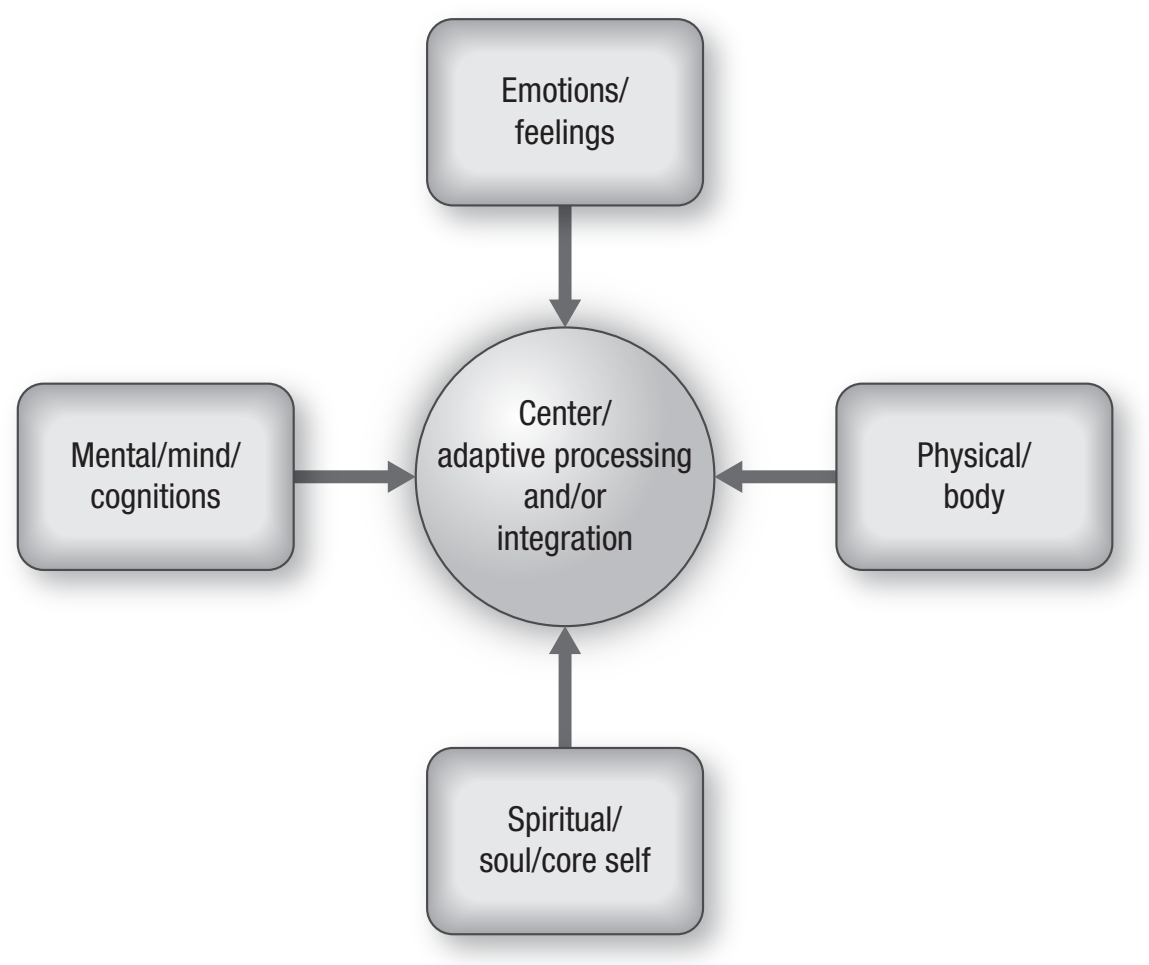

FIGURE 1.2 The Dimensions of Sexual Experience.

Source: Adapted from Ogden, G. (2016, May). Exploring the 4-D wheel of sexual health. Workshop presented at the AASECT Northeast Region Meet and Greet. Florence, MA.

with divine forces, experiencing oneself as part of all that is sacred. It can be characterized by ecstasy, increased energy, lasting satisfaction, connection, and transcendence. The shadow side can include a profound sense of isolation, depression, and paralysis (Ogden, 2016).

The Center of these four quadrants converging positively varies by individual. Some folks may report that visiting this center feels like a "high-definition Oz." The center "may feel like place of mystery and paradox where opposites emerge in an uncanny way." The person "may experience one-ness, integration, shape-shifting, timelessness, light, and/or lightness of being." They may report communing "profoundly" with self and/or partner. The center may feel like a "place of clarity, vision, vastness, and unconditional love." As with the four quadrants, the center has a shadow side: hopelessness and despair (Ogden, 2016).

You can have your client explore and address an issue or potential EMDR target using the four dimensions of the wheel. As with EMDR, you will want to create 
a sense of safety in the office, perhaps in one specific area (or one specific area in their home, if you are doing teletherapy). Invite your client to try a movement to address the issue. Help your client develop a ritual. If possible, you may want to apply slow bilateral stimulation (BLS). Concretize abstract concepts with tangible objects (feathers, rocks, shields, pillows, etc.) and have the client hold them while receiving the BLS and holding positive cognitions related to the concept.

Please refer to Ogden's 2018 book Expanding the Practice of Sex Therapy for full instruction on incorporating her 4-D Wheel into your client work. Consider completing the 4-D training and joining the 4-D network.

\section{INTEGRATING SEXUAL HEALTH INTO THE EMDR ADAPTIVE INFORMATION PROCESSING MODEL}

When Francine Shapiro began developing EMDR therapy in the late 1980s, she initially posited a theoretical model called "Accelerated Information Processing" to explain the framework and efficacy of EMDR therapy. Over time the name evolved to Adaptive Information Processing (AIP) while retaining many of the original hypotheses and principles (Shapiro, 2018). The main tenets of the AIP model focus on healing using the client's resources and innate ability to process maladaptive information to complete integration. Ideally a human brain can usually process information satisfactorily, filing that data away appropriately and neatly, like in a file cabinet. However, if this "information system is impaired, the memory will be stored in a raw, unprocessed, and maladaptive form" (Hase et al., 2017).

This means that this disturbing event will have difficulty connecting to other memory networks that hold adaptive information. Therefore, "when a memory is encoded in such excitatory, state-specific form, the original perceptions can be triggered by a variety of internal and external stimuli" (Hase et al., 2017). These dysfunctionally stored memories then form the basis for future maladaptive responses and possible psychopathology. Activating these memories, even years later, can lead to a variety of symptoms from post-traumatic stress disorder (PTSD) to anxiety to "barely noticeable intrusions" (Hase et al., 2017).

I summarized the AIP model to help us understand how a client's sexual health can be negatively impacted at any point in time, leading to sexual unease, discomfort, or dysfunction. From a yet-to-be-born child experiencing domestic violence vicariously through their parent's stress hormones to a child witnessing a parent's sexual coercion to a pre-teen noticing the lack of physical affection from or between parents-these are all events and atmospheres that can negatively impact a client's sexual health. Add in a shaming and sexually punitive society, and it's a miracle that any child can make their way to adulthood with an empowered pleasurable sex life.

EMDR therapy has been an amazing force of good in righting so many wrongs via alleviating suffering and unlocking adaptive cognitions and futures 
for clients, particularly related to sexual traumas. However, we all can do better to look for the thousand tiny cuts our clients have likely sustained from negative messaging around sexuality, and find EMDR targets related to early misinformation, lack of information, shaming of emerging sexuality, and the million other ways our puritanical culture shames individuals for being sexual beings.

Here is a just a small list of potential EMDR targets related to negative sexual socialization or lack of appropriate sexuality education:

1. An adult cismale client reveals he was caught masturbating as a youth and remains mortified.

2. An adult cisfemale client recalls an abusive first boyfriend that criticized her "slutty" outfits in public.

3. An adult discloses first learning about sex through pornographic magazines discovered in the woods, resulting in continued confusion.

4. An adult who went to Catholic primary school mentions how there was no sex education at all. When they began menarche, they were treated with scorn.

5. A gender non-binary adult described how her parents simultaneously shamed her growing interest in dating AND emphasized and enforced feminized notions of beauty (make-up, dresses, body shape, etc.), creating confusion and shame.

Case Study 1.1: Lily: Sexual Trauma, EMDR Therapy, Sexual Health, Sexual Rights and Values, PLISSIT, Consent, 4-D Wheel

The following case composite example draws from many clients for the purpose of illustrating the aforementioned sexual health frameworks, demonstrating how these additional lenses can help an EMDR clinician search for EMDR and sexual health-related targets. Mostly sexual frameworks are touched upon in this example. The following chapters explore the intersection of EMDR therapy and sexual health in greater detail.

Lily sought EMDR therapy to process and treat pre-teen sexual trauma by slightly older neighbors, early college sexual assault, and current sexual dissatisfaction. Lily has a full-time job in Human Resources, is a lesbianidentified ciswoman in her late thirties, is bicultural (Puerto Rican and Irish heritage), a practicing Catholic, and is in a committed long-term monogamous relationship with another ciswoman. She also reported an abortion related to the college sexual assault.

Placing her life experience within the Circles of Sexuality, she experienced Sexualization (unwanted sexual activity), Reproductive Concerns (she continues to harbor shame and guilt about the abortion), Intimacy Concerns (difficulty trusting her partner), and Sensuality Concerns (current 
lack of pleasure with touch, infrequent orgasms, poor body image, and lack of sexual fantasy). She reports comfort with her sexual identity as an out lesbian and ciswoman.

After working on all the obvious EMDR targets of the pre-teen sexual abuse and the college sexual assault, we determined that there were many small trauma ("small t") targets around the negative messaging of sexuality she experienced within her family culture as well as from the religious Catholic culture in which she was raised. We found numerous EMDR targets that rated three or higher on the Subjective Units of Distress scale (SUDs) such as nuns at her Catholic school shaming make-up, her mother's disapproval of her clothing choice, calling her "a slutty dresser," and the neighborhood boys catcalling her (she had developed slightly earlier than her cisfemale peers). She had never received any accurate sex education other than "don't have sex." No one in her family instructed her about menarche; menstrual pads simply showed up in her bathroom drawer one day.

Broadly speaking, she had not been provided with any healthy or accurate sexual education or framework. She had no idea, even through her late twenties, that she was entitled to a "positive and respectful approach to sexuality" (WHO, n.d.), a life free from sexual abuse, nor was she aware of the basic tenets of consent.

Throughout the therapy, Permission (from the PLISSIT) was often given for Lily to discuss her trauma and all of her sexuality concerns and questions. Often Limited Information was provided in the form of sexuality-related self-help resources such as books on women's sexuality. As she had reported some numbing in her genital region, she was referred to a respected sex-positive ob/gyn to rule out any medical condition.

A great many sessions were spent on normalizing sexual health and sexuality, including the right to engage in sexual behavior simply for pleasure and connection. Keeping in mind SIECUS's thirty characteristics of sexually healthy adults, the positive cognitions of "I am normal and can enjoy my body," "I have the right to enjoy and express my sexuality," and "I can identify and ask for what I want" were identified and frequently used. Her negative cognitions included: "Something is wrong with me," "I can't be vulnerable," and "I don't have choice or voice."

Once all these "small t" EMDR targets were addressed, we then used Ogden's 4-D Wheel to identify any lingering difficulties or challenges. Physically Lily continued to report some difficulty identifying arousal and other pleasurable signs. Emotionally she noticed continued fear and shame. Mentally she continued to harbor lingering messages of guilt around sexual enjoyment from her Catholic background, particularly in light of the homophobia she went through in her coming out process. These generated 
even more "small t" appearing targets, which ended up rating higher on the SUDs upon further activation, pushing them into more "big T" territory. Spiritually she reported a lack of deep connection to her body and her partner.

We were able to find more recent and current EMDR targets based on the 4-D Wheel categories. For example, Lily noted that when her partner approached her from behind to hug her, she froze and went numb, essentially shutting down. Once we processed these more current/present targets, we developed and processed several EMDR Future Templates to navigate through any lingering negative experiences of her sexuality.

Additionally, Lily and her partner had joined an Episcopalian "welcoming" congregation a couple of years ago (specifically welcoming to LGBTQ folks). She was now able to parse out her own spiritual identity, retaining the pieces similar to her Catholic upbringing that continued to feel meaningful and positive (her relationship to Jesus and Mary), as well as enjoy a new comfort during church services knowing that she was "normal," loved, and enthusiastically accepted as herself.

At discharge Lily noted consistent sensation perception throughout her body, including her genitals, more orgasms, and a greater interest and ability to receive and enjoy sexual connection with her partner. She was now able to more easily access the "center" of her 4-D Wheel, experiencing timelessness, unconditional love, and integration of Mental, Physical, Emotional, and Spiritual quadrants.

Lily also chose to "install" more resources via BLS including a cherished Celtic cross given to her by a nurturing and open Irish grandmother (representing unconditional love and spiritual connection), shells found in Puerto Rico (representing openness and sensual connection), and her engagement ring from her supportive partner (representing trust, healthy risk-taking, mutual sharing, and pleasure). Lily reported consistently being able to identify and ask for what she wanted, not only sexually, but in her profession, family of origin, and friendships. At the mutual decision of client and therapist, Lily discharged from EMDR therapy with many sex-positive resources and messages, plus full encouragement and support.

\section{SUMMARY}

WHO and the Office of the Surgeon General and Office of Population Affairs both have helpful definitions of sexual health. A working definition of sexual activity is provided, and the uplifting and affirming WAS's Declaration of Sexual Rights and Pleasure is shared. 
Many individual and cultural factors (e.g., erotophobia) impact therapists' potential comfort and discomfort in addressing sexual health with their clients. Therapists also bring their own Sexological Worldview into the session; therefore, gaining knowledge and familiarity with sexual health is critical. There are many ways therapists can increase their comfort with sexual material, including training such as a SAR workshop.

The concept of consent (particularly FRIES) is extremely important, not only regarding clients' experiences with sexual activity, but for ourselves as therapists as we inquire about our clients' sexual health. The PLISSIT model is an excellent model for sexual health communication and intervention.

The Our Whole Lives and the Planned Parenthood curricula embrace many sex positive values (e.g., dignity and self-worth, knowledge, choice, equal rights and value), which we can keep in mind in our work with clients. Gina Ogden's 4-D Wheel of Sexuality (Mental, Physical, Emotional, Spiritual, and Center dimensions) also offers a helpful clinical framework and is easily integrated into the AIP Model.

\section{REFERENCES}

Advocates for Youth. (1995). Life planning education: A youth development program. Author.

Annon, J. S. (1976). The PLISSIT model: A proposed conceptual scheme for the behavioral treatment of sexual problems. Journal of Sex Education and Therapy, 2(1), 1-15. https:// doi.org/10.1080/01614576.1976.11074483

Bright, S. (1997). Sexual state of the union. Touchstone.

Bruess, C., \& Schroeder, E. (2018). Sexuality education: Theory and practice, seventh edition. ETR.

Buehler, S. (2017). What every mental health professional needs to know about sex, second edition. Springer Publishing Company.

Dailey, D. (1995). Circles of Sexuality as depicted in life planning education: A youth development program (Dr. Dennis Dailey, DSW, Professor Emeritus, University of Kansas, 1111 East 19th Street, Lawrence, KS 66046-3205).

Dischavio, R. (2016). The deep yes: The lost art of true receiving. Spanda Press.

Gallagher, K. (2014, October). Pandora's Box: Sex from A to Z in clinical work [Presentation]. Brattleboro Retreat, Holyoke, MA.

Global Advisory Board for Sexual Health and Wellbeing. (2016). Working definition of sexual pleasure. https://www.gab-shw.org/our-work/working-definition-of-sexual-pleasure/

Gruskin, S., Yadav, V., Castellanos-Usigli, A., Khizanishvili, G., \& Kismödi, E. (2019). Sexual health, sexual rights and sexual pleasure: Meaningfully engaging the perfect triangle. Sexual and Reproductive Health Matters, 27(1), 29-40. https://doi.org/10.108 0/26410397.2019.1593787

Hase, M., Balmaceda, U., Ostacoli, L., Liebermann, P., \& Hofmann, A. (2017). The AIP model of EMDR therapy and pathogenic memories. Frontiers in Psychology, 21. https:// doi.org/10.3389/fpsyg.2017.01578 
Klein, M. (2010, April). When sex gets complicated: Pornography, kink, cybersex, and other clinical challenges [Workshop]. AASECT, Florence, MA.

Kleinplatz, P. J., Menard, A. D., Paquet, M.-P., Paradis, N., Campbell, M., Zuccarino, D., Mehak, L. (2009). The components of optimal sexuality: A portrait of "great" sex. The Canadian Journal of Human Sexuality, 18(1-2).

Mexico City World Congress of Sexual Health. (October 15, 2019). Declaration on Sexual Pleasure. https://worldsexualhealth.net/declaration-on-sexual-pleasure/

National Center for Injury Prevention and Control, Division of Violence Prevention. (2021, February 5). Preventing sexual violence. Centers for Disease Control and Prevention. https://www.cdc.gov/violenceprevention/sexualviolence/fastfact.html

Office of the Surgeon General \& Office of Population Affairs. (2001). The Surgeon General's call to action to promote sexual health and responsible sexual behavior. Office of the Surgeon General. htto://www.ncbi.nlm.nih.gov/NBK44216

Ogden, G. (2016, May). Exploring the 4-D wheel of sexual health. Workshop presented at the AASECT Northeast Region Meet and Greet, Florence, MA.

Ogden, G. (2018). Expanding the practice of sex therapy: The Neuro-update edition - An integrative approach for exploring desire and intimacy (2nd ed.). Routledge.

Queen, C. (2002). Real-live nude girl: Chronicles of sex-positive culture. Cleis Press.

Saakvitne, K. W., Pearlman, L. A., \& Staff. (1996). Transforming the pain: A workbook on vicarious traumatization. W.W. Norton \& Company, Inc.

Sexuality Information and Education Council of the United States (2004). Guidelines for comprehensive sexuality education, $3^{\text {rd }}$ edition: Kindergarten through $12^{\text {th }}$ grade. National Guidelines Task Force.

Shapiro, F. (2018). Eye movement desensitization and reprocessing therapy: Basic principles, protocols, and procedures, third edition. Guilford.

Sitron, J. A., \& Dyson, D. A. (2012, March 13). Validation of sexological worldview: A construct for the use in the training of sexologists in sexual diversity. SAGE Open. https://doi.org/10.1177/2158244012439072

South Shore Sexual Health Center. (n.d.). Retrieved September 23, 2021, from https:// www.sssexualhealthcenter.com/getcertified\#sar

United Nations Educational, Scientific and Cultural Organizations. (2018). International technical guidance on sexuality education. UNESCO Joint United Nations Programme on HIV/AIDS, United Nations Populations Fund, United Nations Children's Fund, United Nations Entity for Gender Equality and the Empowerment of Women, World Health Organization.

Van der Hart, O., Nijenhuis, E. R. S., \& Steele, K. (2006). The haunted self: Structural dissociation and the treatment of chronic traumatization. W.W. Norton and Company.

Westheimer, R., Grunebaum, A., \& Lehu, P. (2012). Sexually speaking: What every woman needs to know about sexual health. John Wiley and Sons.

Wilson, P. (2014). Our whole lives: Sexual education for grades 7-9, 2nd edition. UUAC.

World Health Organization. (n.d.). Sexual and reproductive health. Retrieved September 23, 2021, from http://www.who.int/reproductivehealth/topics/sexual_health/sh_definitions/en 


\section{SUPPLEMENTAL MATERIALS FOR CLIENTS}

I have included here a helpful article I wrote for my monthly column, Sex Matters, that can be handed out to clients to further explain consent and pleasure.

\section{Consent and Pleasure}

By Stephanie Baird, LMHC

(Originally published December 2019 in the Montague Reporter newspaper monthly Sex Matters column.)

Let's talk about consent and pleasure. As someone who came of age in the eighties and nineties, not much was discussed or even known about active negotiation of sex-related activities. During my work in the late nineties, primarily working with sexual assault survivors, "consent" concepts emerged in clinical work as well in rape crisis educational campaigns. However, most of these campaigns centered around the "no means no" concept. This, of course, is paramount. Humans being able to say no to unwanted activities is the foundation from which we can later say yes to wanted activities.

Much of the twentieth century stereotypical male and female gender expression involved the idea that a cisgender man should want and try for sex, and that a "good" cisgender woman should be a "gatekeeper," saying no often, but eventually "giving in" (like Scarlet O-Hara and Rhett Butler in "Gone With the Wind," he forcibly dragged her, kicking and fighting, upstairs for sex, and then the next scene shows her post-sex, looking content). It wasn't ok for a woman to outright want and ask for sexual activity - that desire somehow put the woman in a "slut" category. Happily our wonderful twenty-first century has brought increased activism with folks naming "slut-shaming" as a bad thing, along with the creation of the problematic term "man-slut" (which I dislike), in an attempt to equalize notions of sexual desire.

Now that we are one-fifth into the twenty-first century, with \#metoo solidly in place, humans continue to expand the conversation around consent. At a minimum, for a person to legally consent to any sexual activity (physical, verbal, or otherwise), the person needs to be of age (16 is the youngest age for any type of intercourse in Massachusetts, this varies state by state).

Consent has further evolved into the acronym FRIES. According to Planned Parenthood, modern full consent includes

1. Freely given. "Consenting is a choice you make without pressure, manipulation, or under influence of drugs or alcohol."

2. Reversible. "Anyone can change their mind about what they feel like doing, anytime. Even if you've done it before," and you are preparing to do it again. 
3. Informed. "You can only consent if you have the full story. For example, if someone says they'll use a condom, but don't, then there isn't full consent."

4. Enthusiastic. "When it comes to sex, you should only do things you WANT to do, not things you are expected to do."

5. Specific. "Saying yes to one thing (like going to the bedroom to make out) doesn't mean you've said yes to other" things (like intercourse).

I personally love the addition of enthusiasm, as this concept finally equalizes desire within all the genders. No longer does sex just have to be a dude being trained all his life to seize a moment of sex whenever opportunity is there. Now women and other genders have been given permission, and the imperative, to enthusiastically ask for what they want.

In November 2019 I heard acclaimed journalist Peggy Orenstein speak on "Girls and Sex: From Risk and Danger to Responsibility and Joy" (Boston), and learned some helpful terms and alarming statistics.

She noted that research psychologist Sarah MacLelland coined the term "'intimate justice': the idea that sex has political as well as personal components, reflecting issues of gender inequality, economic disparity, violence, and mental health. Intimate justice asks: Who is entitled to engage in a sexual experience? Who is entitled to enjoy it? How does each partner define good enough?"

Orenstein shared research that looked at cisfemale self-objectification (girls trying to attain an "effortless perfection" of "hotness and sexiness" for the "male gaze") and found that higher self-objectification correlated with reduced sexual satisfaction among cisfemales. It is also linked with depression, anxiety, eating disorders, negative and reduced cognition, and political participation. She noted that many heterosexual teens and young women seemed to undergo a psychological cliterodectomy, preferring to give rather than receive any kind of pleasure, mostly for status, security, and notions of power. However, the "orgasm gap" disappears among lesbians.

One antidote for this lopsided quandary of who gets to receive pleasure can be found in an encouraging book by local author Dr. Rosalyn Dischiavo called The Deep Yes: The Lost Art of True Receiving (2016). At a talk she gave, one of the first things Dischiavo asked was if we remembered our last shower. She pointed out that folks often mindlessly rush through their showers, sometimes even forgetting if they have shampooed, when showering is a great opportunity to say yes to mindful sensuality with oneself.

Her book explores the original yin energy of receptivity as one of "drawing in ... allowing," and incredibly active in its "power to receive." In India, "the Goddess Shakti, the embodiment of all power, is the yin principle." Within the white yang of the yin-yang symbol is Spanda (the yang-feminine): spontaneous movement, birthing, and power. Dischiavo applies these ideas to saying yes to sleep, mindful eating, our beautiful bodies, and pleasurable sex with oneself and others. 
After hearing Orenstein's alarming review of the pleasure disparity, and her mention that even modern sex educators fail to mention clitoris to their children at early ages, I found a diagram of female genitalia that included the clitoris and gave my nearly 9-year-old daughter a quick clitoris lesson, telling her that it's the only organ whose sole purpose seems to be fun and pleasure. Her eyes widened with interest as she said, "you mean it's something that's supposed to feel good and it's a good thing to touch it?" I answered with a resounding "yes" (and advised her that alone time in her bedroom is a great way to explore). 


\section{DECLARATION OF SEXUAL RIGHTS, PLEASURE, AND CONSENT HANDOUT}

Since 1978 the World Association for Sexual Health (WAS) has worked toward sexual health and rights. Thousands of members attend global biennial congresses and in recent years have made declarations for sexual rights.

\section{Declaration of Sexual Rights (Adapted from WAS, 2014)}

1. The right to equality and freedom of sexual expression.

2. The right to make decisions about one's body in regard to sexuality.

3. The right to be safe from all forms of sexual violence and coercion, and be free from inhumane treatment or punishment because of one's sexual or gender expression.

4. The right to accurate scientific information and education about sexuality and sexual health.

5. The right to freely create and dissolve marital and other similar relationships.

6. The right to family planning resources.

7. The right to organize freely to promote sexual rights and health.

8. The right to privacy regarding sexual matters.

9. The right to judicious laws and remedies regarding sexual rights.

10. The right to the highest attainable standard of sexual health, including sexual safe and pleasurable sexual experiences.

[Source: Adapted from the World Association for Sexual Health. (2014, March). Declaration of Sexual Rights. https://worldsexualhealth.net/wp-content/ uploads/2013/08/declaration_of_sexual_rights_sep03_2014.pdf.]

\section{Declaration on Sexual Pleasure}

Sexual pleasure is the physical and/or psychological satisfaction and enjoyment derived from shared or solitary erotic experiences, including thoughts, dreams, and autoeroticism. Self-determination, consent, safety, privacy, confidence...communicating and negotiating sexual relations are key...factors for pleasure to contribute to sexual health and well-being. Sexual pleasure should be exercised within the context of sexual rights... (including) equality, non-discrimination, autonomy, bodily integrity, and the right to the highest...standard of health and freedom of expression. The experiences of human sexual pleasure are diverse and sexual rights ensure that pleasure is a positive experience for all concerned and not obtained by violating...human rights and well-being. (Gruskin et al., 2019) 
Some of WAS's Declaration of Sexual Rights include:

- Sexual pleasure is a fundamental part of sexual and human rights and includes the possibility of diverse sexual experiences.

- Access to appropriate sources of sexual pleasure is part of human well-being.

- Sexual health and well-being include pleasurable and safe sexual experiences free of discrimination, coercion, and violence.

- Sexual pleasure shall be globally integrated into education, health services, research, and advocacy and contributes to global health.

\section{Consent}

According to the Planned Parenthood website, modern full FRIES consent is:

1. Freely given. "Consenting is a choice you make without pressure, manipulation, or under influence of drugs or alcohol."

2. Reversible. "Anyone can change their mind about what they feel like doing, anytime. Even if you've done it before," and you are preparing to do it again.

3. Informed. "You can only consent if you have the full story. For example, if someone says they'll use a condom, but don't, then there isn't full consent."

4. Enthusiastic. "When it comes to sex, you should only do things you WANT to do, not things you are expected to do."

5. Specific. "Saying yes to one thing (like going to the bedroom to make out) doesn't mean you've said yes to other" things (like intercourse). 
